\title{
Simultaneous CARS and Interferometric Rayleigh Scattering
}

\author{
Daniel Bivolaru*, Paul M. Danehy ${ }^{\dagger}$, Keith D. Grinstead, Jr. ${ }^{\S}$, Sarah Tedder ${ }^{\ddagger}$, and Andrew D. Cutler"
}

NASA Langley Research Center, Hampton VA, 23681-2199

\begin{abstract}
This paper reports for the first time the combination of a dual-pump coherent antiStokes Raman scattering system with an interferometric Rayleigh scattering system (CARS IRS) to provide time-resolved simultaneous measurement of multiple properties in combustion flows. The system uses spectrally narrow green (seeded Nd:YAG at $532 \mathbf{n m}$ ) and yellow $(552.9 \mathrm{~nm})$ pump beams and a spectrally-broad red $(607 \mathrm{~nm})$ beam as the Stokes beam. A spectrometer and a planar Fabry-Perot interferometer used in the imaging mode are used to record the spectrally broad CARS spectra and the spontaneous Rayleigh scattering spectra, respectively. Time-resolved simultaneous measurement of temperature, absolute mole fractions of $\mathrm{N}_{2}, \mathrm{O}_{2}$, and $\mathrm{H}_{2}$, and two components of velocity in a Hencken burner flame were performed to demonstrate the technique.
\end{abstract}

\section{Introduction}

$\mathrm{D}$ esign of the next generation of aerospace vehicles will rely heavily on computational fluid dynamics (CFD) simulations. However, nonintrusive, quantitative diagnostic techniques are needed to validate the CFD codes for accuracy and are necessary both for ground testing and in-flight measurements. It is desirable to obtain timefrozen single-point measurements so that unsteady and turbulent features of the flow can be investigated. For example, dual-pump coherent anti-Stokes Raman scattering (CARS) has been used to obtain temperature and species mole fraction in high speed combusting flows, ${ }^{1,2,3}$ and Rayleigh scattering has been used to obtain temperature, density and velocity in high speed non-reacting flows. ${ }^{4,5,7}$ Measurement of temperature and density in reacting flows using Rayleigh scattering requires knowledge of the gas composition, which can be provided by CARS. CARS and Rayleigh scattering measurements taken independently can provide data for test cases for evaluating CFD codes. When multiple flow properties are measured simultaneously, statistical correlations can be formed which can be used to obtain a deeper understanding of turbulence phenomena in these combusting flows. Correlations can also be used to evaluate parameters in new turbulence models.

There are several techniques that employ Rayleigh scattering to measure velocity. One such technique is Filtered Rayleigh Scattering (FRS). The method analyzes Rayleigh scattering spectra by filtering with an $\mathrm{I}_{2}$ filter, permitting the measurement of velocity, temperature, and density in gas flows of known composition. However, the method has limitations in that the absorption line from the $\mathrm{I}_{2}$ spectrum used for filtering has a narrow bandwidth. Consequently, this approach has a limited velocity dynamic range and does not work at high temperatures ( $>1000$ $\mathrm{K}$ ) where the Rayleigh spectrum is much broader than that of the $\mathrm{I}_{2}$ absorption line. Furthermore, the method requires a stabilized laser frequency and a calibration curve to measure velocity. The technique does not work if Mie scattering from particles contaminates the Rayleigh scattering. The biggest advantage of this approach is that it can be used for instantaneously mapping a two-dimensional flow field.

A second approach that permits a larger dynamic range of measurable velocities, and works at low ${ }^{6,7,8}$ as well as at high ${ }^{5}$ temperatures, is the interferometric Rayleigh scattering method. This technique has previously been used for single-point velocity, density, and temperature measurements. Planar Fabry-Perot and confocal etalons were used for Rayleigh signal analysis in conjunction with either cooled or intensified CCD cameras, or photo-multiplier

\footnotetext{
* NASA Postdoctoral Fellow, Advanced Sensing and Optical Measurement Branch, MS 493, AIAA Member

${ }^{\dagger}$ Research Scientist, Advanced Sensing and Optical Measurement Branch, MS 493, AIAA Associate Fellow

${ }^{\S}$ Research Scientist, Swales Aerospace, AIAA Member

${ }^{\ddagger}$ Research Assistant, College of William and Mary, Williamsburg, VA 23669, AIAA Member

" Professor, The George Washington University, Newport News, VA 23602, AIAA Associate Fellow
} 
arrays to image the interferogram., $5,6,8,9,10,11,12$ The shape of the Rayleigh scattering spectrum is a function of the parameter $\boldsymbol{y}=\alpha \mathbf{K} a$, where $\alpha=p / \mu$ is the collision frequency ( $p$ is the gas pressure and $\mu$ is the shear viscosity) and $\mathbf{K} a$ is the acoustic spatial frequency ( $\mathbf{K}$ is the wave vector and $a$ is the speed of sound). For low density gases and high temperatures, when the parameter $\boldsymbol{y}<<1$, the Rayleigh spectrum can be approximated with a Gaussian shaped spectrum. The peak of the spectrum is Doppler shifted by a frequency proportional to the bulk velocity, the spectral width is proportional to the square root of the gas temperature, and its amplitude is proportional to the gas density. To obtain these gas parameters, the Rayleigh spectrum is analyzed by applying a fitting technique to the experimental data using the laser spectrum as reference ${ }^{8}$ or by correlation. ${ }^{7}$

In this paper we present for the first time the combination of a dual-pump CARS system with an interferometric Rayleigh scattering system (CARS-IRS) to provide time-resolved simultaneous measurement of multiple flow properties including velocity.

\section{Instrument Configuration}

\section{A. Experimental Arrangement}

The experimental arrangement of the combined system is shown in Fig. 1. For the measurement of temperature and the absolute mole fractions of $\mathrm{N}_{2}, \mathrm{O}_{2}$, and $\mathrm{H}_{2}$ we use a dual-pump CARS method. ${ }^{1,2,3}$ The system uses spectrally-narrow green (injection seeded Nd:YAG at $532 \mathrm{~nm}$ ) and yellow (NB Dye laser at $552.9 \mathrm{~nm}$ ) laser pump beams and one spectrally broad red laser (BB Dye laser at $607 \mathrm{~nm}$ ) beam as the Stokes beam. The $850 \mathrm{~mJ} / \mathrm{pulse}$ energy of the Nd:YAG laser is split in four ways. About $350 \mathrm{~mJ}$ of the energy is used for the CARS pump beam and for Rayleigh scattering. Another $250 \mathrm{~mJ}$ is used for pumping a homemade broadband modeless ${ }^{13}$ dye laser at 607 $\mathrm{nm}$ that uses Rhodamine 640 laser dye diluted in methanol. The rest of the Nd:YAG laser energy is used to pump a tunable narrowband dye laser at $552.9 \mathrm{~nm}$ (Quantel TDL-51).

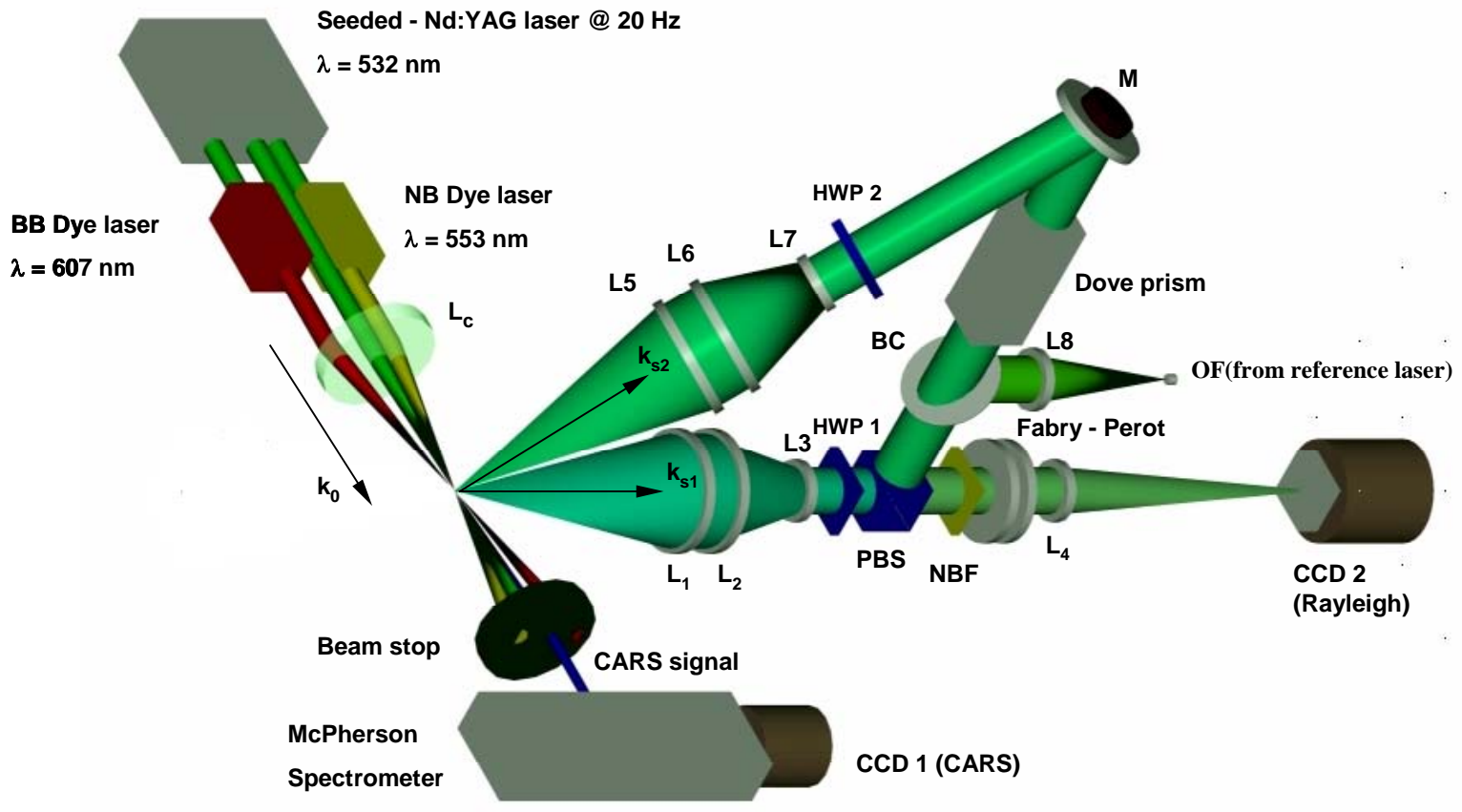

Figure 1. Experimental setup of the combined CARS - IRS system. 
The beams are combined at the focusing point of a spherical lens $L_{c}$ (focal length of 410 $\mathrm{mm}$ ) in a folded BOXCARS geometry ${ }^{14}$ generating a measurement volume of about 1.5 $\mathrm{mm} \times 0.2 \mathrm{~mm} \times 0.2 \mathrm{~mm}$. The frequency difference between the green and red beams corresponds to the vibrational Raman shift of $\mathrm{N}_{2}$. The frequency of the yellow pump beam is chosen so that the frequency difference between the yellow and red beams equals the vibrational Raman shift of $\mathrm{O}_{2}$. Pure rotational $\mathrm{H}_{2}$ transitions are also present in both spectral regions. The input beams plus the coherent blue signal beam at $491 \mathrm{~nm}$ are collected and collimated by another spherical lens with the same focal length as Lc. The three input beams are captured in a beam dump while the blue signal beam is passed through. After passing through an interference filter to reject unwanted light, the signal beam is focused by two "crossed" cylindrical lenses on the input slit of a one-meter spectrometer (McPherson) with a 1200 groove/mm grating. The CARS signal, which is a spectrally broad blue beam that contains $\mathrm{N}_{2}, \mathrm{O}_{2}$ and $\mathrm{H}_{2}$ spectra, is analyzed by a spectrometer and recorded by the

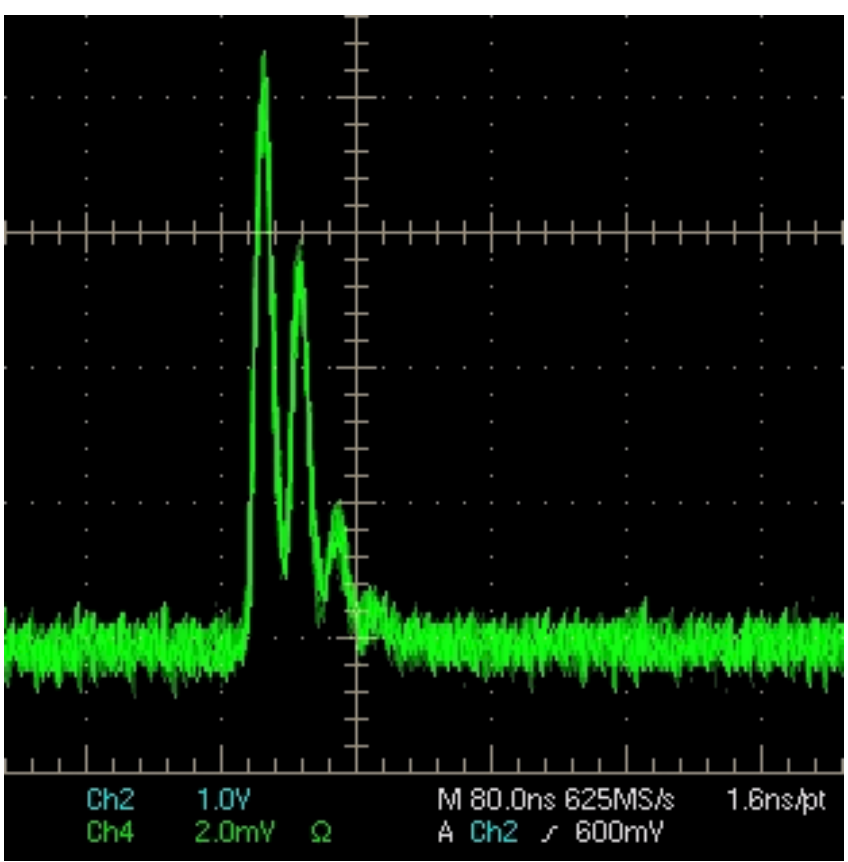

Figure 2. The variation of the laser pulse energy in time.

CCD1 camera (Princeton Instruments PIXIS-100B with an area of $1340 \times 100$ pixels). The shape of these spectra provides information on the temperature while the relative intensities of these spectra provide a measure of the relative mole fractions. The spectrum is fit with a theoretical model to determine the temperature and mole fractions.

The velocity measurement is performed simultaneously using an interferometric Rayleigh scattering measurement system, earlier versions of which are described in References 8 and 11 . The same pulsed seeded green laser beam employed in the CARS technique is used as a narrow-band light source for the Rayleigh scattering technique. The breakdown of the gas at room temperature limited the laser pulse energy at $65-75 \mathrm{~mJ} / \mathrm{pulse}$. To avoid this problem, and to increase the laser energy provided for the Rayleigh scattering system, a fraction of the laser energy of this beam (60\%) was delayed 18 nsec and re-injected on the same optical axis by using a beam splitter. The optical arrangement is similar to the double-delay line pulse stretcher described in Ref. 8 except for removing the output beam combiner. The single-delay line method was chosen for this experiment because it proved to be more mechanically stable and easier to align compared to the double-delay line while having similar overall efficiency. At full input laser power, the pulse stretcher can deliver a total of $245 \mathrm{~mJ}$ in three pulses with an efficiency of about $70 \%$. Only about $150 \mathrm{~mJ}$ (green), $9 \mathrm{~mJ}$ (yellow), and $6 \mathrm{~mJ}$ (red) of of the Nd:YAG laser energy are delivered to the probing volume due to losses in the transmission and combining optics. The output beam pulse temporal shape of the green beam is shown in Fig. 2.

The receiving optics for Rayleigh are designed to capture the Rayleigh scattered light from the green pump beam in the measurement volume while preserving the scattering angle information, and to mix it together with the unshifted light of the laser before it is passed through the interferometer. In this way more than one component of velocity can be measured in one interferogram. ${ }^{8}$ Reference 8 shows several different approaches for combining Rayleigh signals to determine two or three velocity components simultaneously at multiple closely-spaced points. The vertically polarized laser beam with the wave vector $\boldsymbol{k}_{0}$ is focused in a small region at the measuring location. The scattered light collected in the direction of $\boldsymbol{k}_{s 1}$ has a Doppler shift frequency given by $\Delta v_{1}=\left(\boldsymbol{k}_{s 1}-\boldsymbol{k}_{0}\right) \cdot \boldsymbol{V}$, where the $\boldsymbol{k}_{\mathrm{s} 1}$ is the wave vector of the scattered light and $\boldsymbol{V}$ is the velocity vector. The direction of the $\left(\boldsymbol{k}_{s 1}-\boldsymbol{k}_{0}\right)$ vector is the direction of the velocity component, defined as $V_{1}$, Given the orientation of this vector relative to the burner surface, this velocity vector is the radial component of velocity, $V_{r}$. The Rayleigh scattered light in the $\boldsymbol{k}_{s 1}$ direction is collected and collimated by the $\mathrm{L}_{1}$ lens. The beam diameter is reduced to match the interferometer's inputaperture using lenses $\mathrm{L}_{2}$ and $\mathrm{L}_{3}$. A half wave plate (HWP 1) adjusts the polarization to vertical and the beam is sent through the polarization dependent beam combiner (PBS) towards the interferometer (Fabry-Perot).

To measure the second component of velocity $V_{z}$ in the axial direction of the flow issuing from the burner, scattered light is collected in the direction of the wave vector $\boldsymbol{k}_{\mathrm{s} 2}$ in a plane (containing $\boldsymbol{k}_{\mathrm{s} 1}$ ) perpendicular to the 
plane defined by the vectors $\boldsymbol{k}_{s 1}$ and $\boldsymbol{k}_{0}$. The angle between $\boldsymbol{k}_{s 2}$ and $\boldsymbol{k}_{s 1}$ is $33^{\circ}$. The direction of $\left(\boldsymbol{k}_{s 2}-\boldsymbol{k}_{0}\right)$ vector gives the direction of the velocity vector being measured, defined as $V_{2}$. This vector contains information from both components of velocity $V_{r}$ and $V_{z}$. The light is collected and collimated by the lens $\mathrm{L}_{5}$, while the lenses $\mathrm{L}_{6}$ and $\mathrm{L}_{7}$ reduce the beam diameter. After the half wave plate HWP 2 rotates the beam polarization to horizontal, the mirror M reflects the collimated beam toward the polarization dependent beam combiner PBS. A Dove prism is used to rotate the laser beam image with an angle that is convenient for image processing (usually $90^{\circ}$ ). A small portion of the light reflected from one of the pulse forming optics is collected by an optical fiber (OF) to be used as a frequency reference. Two cross-polarizers are used to adjust the intensity of this reference light. Although the intensity of the reference laser light must be monitored carefully to be of the order of the received scattered light intensity, the system does not require special devices for filtering out this radiation. Thereafter, the reference laser light is mixed together with the Rayleigh scattered light using the beam combiner BC (a glass plate). The combined beam is filtered out to remove the yellow and the red CARS beams using a better than $90 \%$ transmission narrow band pass filter (NBF) at $532 \mathrm{~nm}$ before it is passed through the interferometer. An electron multiplication gain CCD camera (CCD2 - Princeton Instruments PHOTON Max 512 with an area of $512 \times 512$ pixels) images the interference fringe pattern, which is formed at the output of the interferometer by the lens $\mathrm{L}_{4}$ and contains both the signal from two collecting directions and the reference frequency information.

In the example interferogram obtained with this system, shown in Fig. 3, each of the horizontal and vertical high intensity fringe patterns in the image plane represents a measurement direction. The concentric ring pattern is the reference laser frequency. The gas temperature at the measurement volume is $925 \mathrm{~K}$ for frame (a) and $2380 \mathrm{~K}$ for frame (b) which shows a significantly lower intensity of the signal but still measurable. Image processing software is used to extract spectra from each interferogram and analyze for Doppler shift, FWHM, and amplitude of the Rayleigh spectra.

The air-gap adjustable planar Fabry-Perot interferometer used here was set for a free spectral range (FSR) of $7.5 \mathrm{GHz}$. This gives a range of measurable velocities from 0 to $\sim 3 \mathrm{~km} / \mathrm{sec}$ up to temperatures of about $2500 \mathrm{~K}$. If the magnitude of the velocity of interest had only positive or only negative values, as for example in the case of axial velocity, the instrument could use the full FSR for measurement without ambiguity. In regions where the velocity can take either sign, only half of the FSR (on each side of zero) can be used for measurement without ambiguity.

Both, the CARS spectra and the Rayleigh spectra are acquired simultaneously by synchronizing the cameras with the green laser qswitch pulse at $20 \mathrm{~Hz}$.

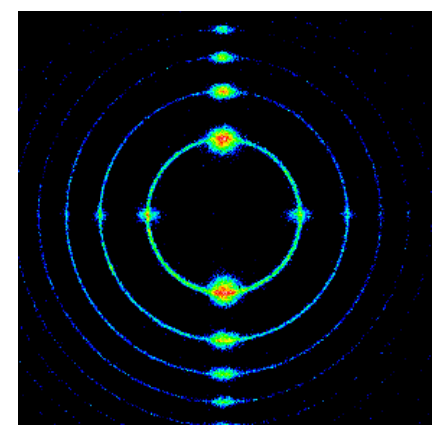

(a)

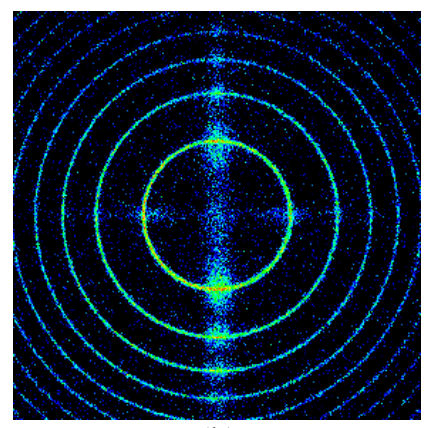

(b)

Figure 3. Single-shot Fabry-Perot interferograms of Rayleigh scattering at (a) $925 \mathrm{~K}$ and (b) $2380 \mathrm{~K}$, containing spectral information to solve for two components of velocity, and gas temperature and density knowing the gas composition from CARS.

\section{B. Spectra Processing}

The background is subtracted from the CARS signal to correct for camera dark noise and other light not originating from the CARS signal. The background corrected spectra is then divided by a fit of an averaged "nonresonant" spectrum to correct for the shape of the broadband dye laser's spectrum. Nonresonant spectra are taken at the beginning or at the end of a data set by flowing Ar at the measurement volume to remove the resonant species of $\mathrm{N}_{2}$ and $\mathrm{O}_{2}$. The broadband dye laser changes its frequency significantly during data collection so a fitting procedure is used to account for this change ${ }^{1}$. Using CARS spectra obtained in room temperature air, an instrument function that accounts for the broadening of the spectrum due to various instrument effects is fitted with a dualGaussian function. The instrument function and other values describing the system are used to create a library of spectra generated by a modified ${ }^{1}$ version of CARSFIT code originally written by Sandia National Laboratories. ${ }^{15}$ The library has entries every $200 \mathrm{~K}$ and a range of species mole fractions with finer grid points in the lower ranges. The fitting algorithm interpolates spectra between the temperature/composition grid points in the library and the result with the smallest residual is selected. For further details of the analysis method see Ref. 2.

Separate processing software is used to extract and analyze the Rayleigh spectra. A simplified version of the method described in Ref. 8 was used for the present calculations. The image processing methodology consists of linearization of the concentric interference patterns from the Fabry-Perot (F-P) interferogram. The important 
variables are the center of the interferogram, the free spectral range of the interferometer, the FWHM, the amplitude and the Doppler shift of the Rayleigh signal. After the center of the interferogram is found, the image is mapped by reading it in $r$ - $\theta$ plane, where $r$ is the radius from the center and $\theta$ is the angle, using the original $(r, \theta)$ coordinate as the new $(x, y)$ coordinate. For small reading angles, $\theta$, the conversion is simplified by averaging a few lines or columns symmetrically either side of a diameter of the interferogram. The fringe spacing is linearized by the F-P interferometer radius relation $R_{p+1}=\left(R_{p}^{2}+2 f^{2} / k\right)^{1 / 2}$, where $R$ is the radius of the fringe, $p$ is the fringe number, $k$ is the order of interference, and $f$ is the focal length of the fringe forming lens. The result of this procedure is to map the fringes from concentric circles into regularly-spaced parallel lines of equal length. The final "linearized" image can be processed directly in 2D by autocorrelation, or by binning the lines in a single average spectrum for each viewing direction and applying fitting techniques. In the present work, a fitting technique using multiple Gaussians, was applied to the linearized spectrum to calculate the spectral range (FSR), the Doppler shift frequency $\Delta f$, the FWHM, and the signal intensity. The velocity components were then calculated based on the experimental geometry using the equation $\Delta f=\left(\mathbf{k}_{\mathrm{s}}-\mathbf{k}_{\mathbf{0}}\right) \cdot \boldsymbol{V} / \lambda$ where, $\lambda$ is the incident laser wavelength, $\boldsymbol{k}_{\mathbf{0}}$ and $\boldsymbol{k}_{\boldsymbol{s}}$ are the incident and the scattered wave vectors, and $\boldsymbol{V}$ is the gas velocity.

\section{Results and Discussion}

Experiments to demonstrate the combined technique were carried out in a Hencken burner, which provides an adiabatic flat $\mathrm{H}_{2}$-air atmospheric pressure flame, at NASA Langley Research Center. The burner was mounted on a 3D stage, allowing axial and radial scans of the flame through the fixed measurement volume location at the maximum speed of $0.55 \mathrm{~mm} / \mathrm{sec}$ in increments of $0.1 \mathrm{~mm}$. The flame was stabilized with a co-flow of $\mathrm{N}_{2}$. Experiments were performed for a range of equivalence ratios up to 2 . Only data for low equivalence ratios were

(a)

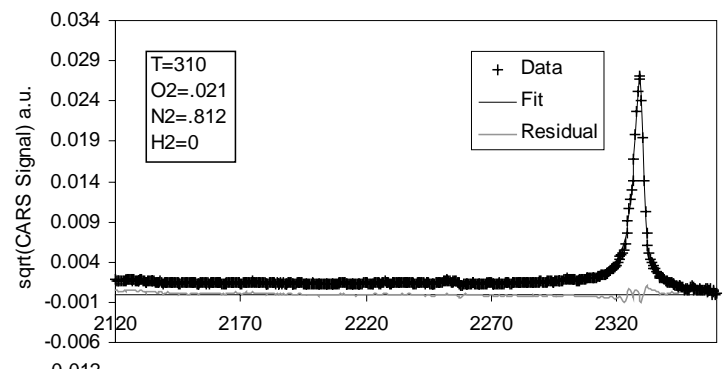

(b)

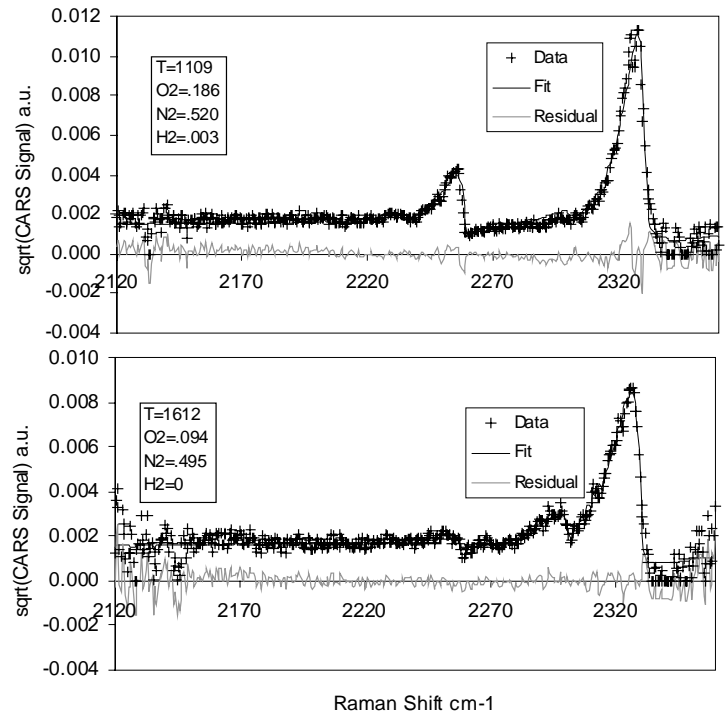

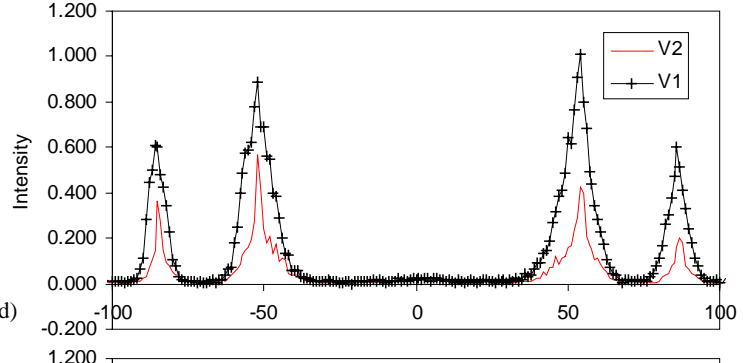

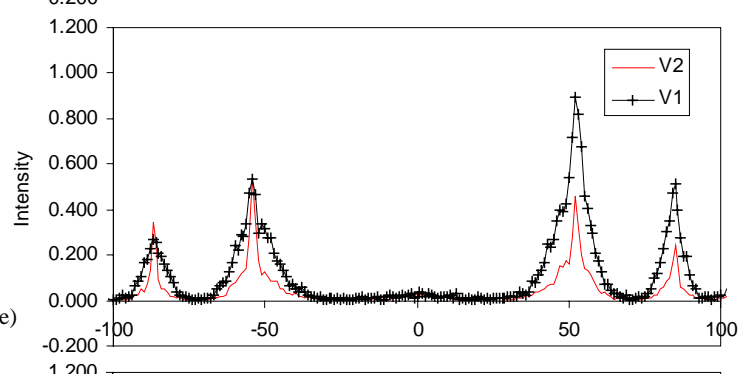

(e)



Figure 4. Simultaneous spectra of CARS (left) and Rayleigh scattering from two viewing directions (right). The CARS spectra are both data and fits of theory to the data. The Rayleigh spectra are data points connected by lines. 
processed and are presented in this paper. The CARS system probed a volume of about $1.5 \mathrm{~mm}$ length and $0.2 \mathrm{~mm}$ diameter perpendicular to the flow direction. The spatial resolution of each measurement point of the Rayleigh signal was about $0.2 \times 0.2 \times 0.2 \mathrm{~mm}^{3}$. Due to large laser scattering from the burner surface the minimum distance from the nozzle exit where measurements could be made was about $8.5 \mathrm{~mm}$ from the exit plane. Since the interferogram contains spatial as well as spectral information, the separation distance between the first two consecutive fringe orders on one side of the interferogram (one FSR) corresponds to about 0.2 $\mathrm{mm}$ in the flow and the diameter of the first order fringe to about $0.5 \mathrm{~mm}$ in the flow. The goal of this experiment was to acquire both CARS and Rayleigh spectra at higher temperatures in a single laser shot and perform a preliminary characterization of the system.

Simultaneous CARS and Rayleigh scattering spectra up to $1610 \mathrm{~K}$ are shown in Fig. 4 (a-c) and in Fig. 4 (d-f), respectively: (a) and (d) are in the coflow of $\mathrm{N}_{2}$, (b) and (e) are in a high temperature flow containing $\mathrm{N}_{2}$ and $\mathrm{O}_{2}$, and (c) and (f) are in a high temperature flow containing $\mathrm{N}_{2}$ and a low proportion of $\mathrm{O}_{2}$. The CARS spectral plots show the experimental data, the theoretical fits, and also the residual between them. The larger residual at higher temperatures is attributed to the broadband dye laser spectrum fluctuations in frequency and shape from shot to shot, which cannot be accounted for, and photon shot noise. These spectra were used to calculate the vibrational gas temperature and the mole fraction of $\mathrm{H}_{2}, \mathrm{O}_{2}$, and $\mathrm{N}_{2}$. They show a good agreement with theory.

The Fabry-Perot interferograms shown in Fig. 3 were obtained during a single laser pulse burst as shown in Fig. 2. Although, the interferogram contains more than 5 fringe orders, only two fringe orders of the spectra are shown in Fig. 4. All four of these fringes could be analyzed for velocity but only one from each spectrumhas been analyzed for this paper, for simplicity. The narrow shaped peak, more visible in the spectra of higher temperature, is the reference laser frequency (no Doppler shift) and its spectral shape is to a good approximation the interferometer instrument function. Each figure contains two Rayleigh and two reference spectra corresponding to the two collecting directions (that are used to solve for the velocity components, $V_{r}$ and $V_{z}$ ) $V_{1}$ (black trace) and $V_{2}$ (red trace of smaller amplitude). Linearized spectra of reference laser light and Rayleigh scattered laser light of small Doppler shift taken in the flame at $1108 \mathrm{~K}$ are shown in Fig. 5. The dark blue line shows the residual of the fit of two Gaussian functions to the experimental data (one Gaussian function fit to the reference $\mathrm{Gs}_{\text {ref }}$ and one to the Rayleigh scattering $\mathrm{Gs}_{\mathrm{RS}}$ ). Two linearized spectra from consecutive fringe orders are used to determine the free spectral range (FSR) using the reference peaks, and the Doppler shift frequency $\Delta f$, FWHM, and the amplitude for each Rayleigh spectrum. The low

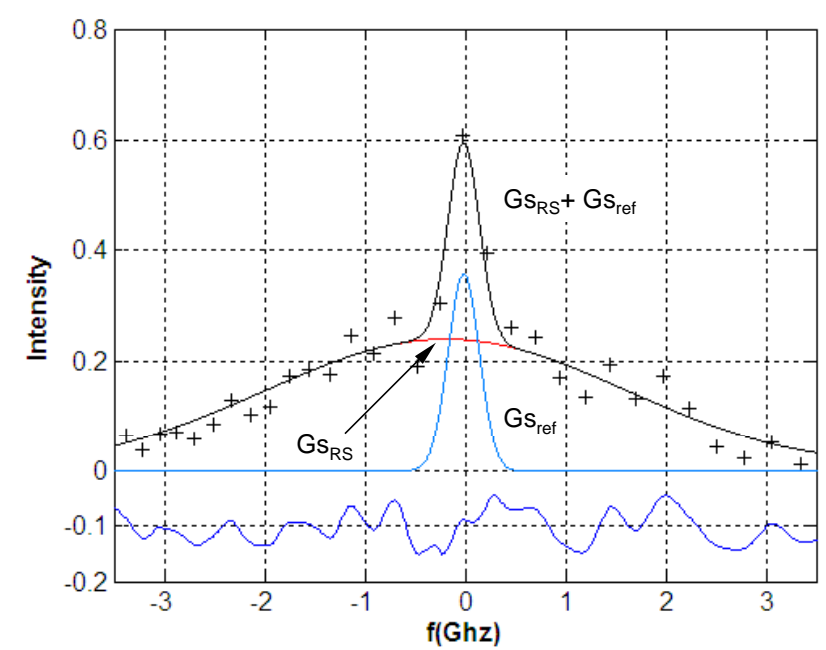

Figure 5. Single shot Rayleigh spectra in $\mathrm{H}_{2}$ - air flame at a temperature of $1108 \mathrm{~K}$. The narrow peak is at the laser frequency.
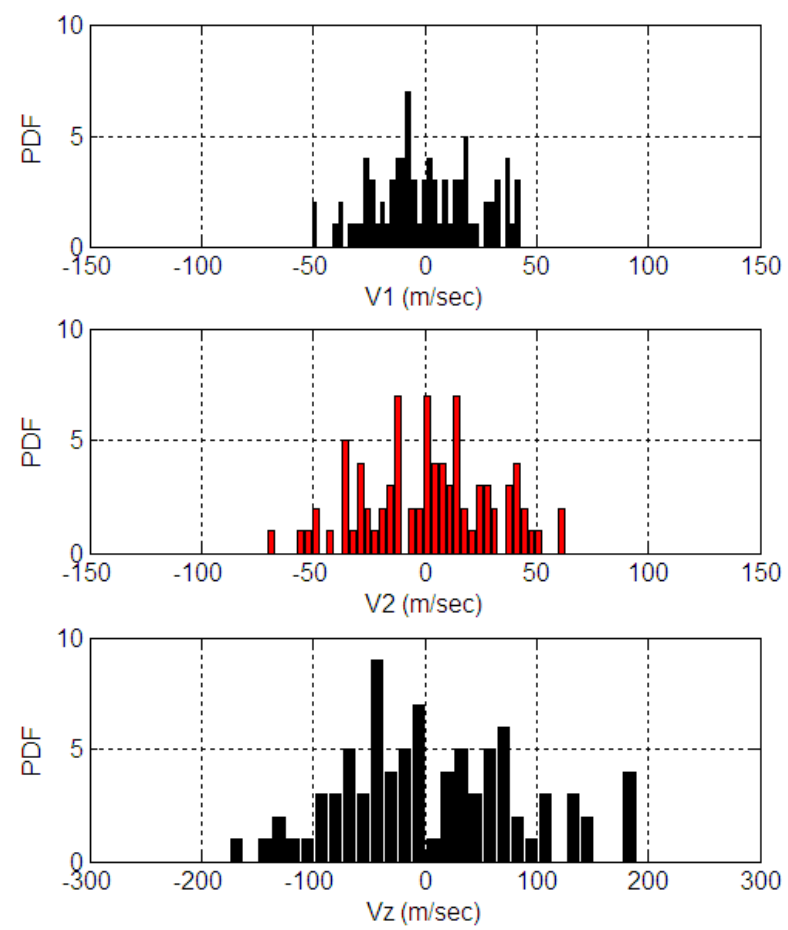

Figure 6. Single shot velocity $\mathrm{PDF}$ in $\mathrm{H}_{2}$ - air flame at an equivalence ratio of $0.1(T=585 \mathrm{~K})$. The average radial velocity is $V r=V_{1}=1 \pm 23 \mathrm{~m} / \mathrm{sec}$, and the calculated axial velocity is $V z=8 \pm 82 \mathrm{~m} / \mathrm{sec}$

6 
amplitude spectrum of the $V_{2}$ collecting direction in Fig. 4 (d), (e), and (f) is attributed to the lower intensity of the Rayleigh scattered light collected in that particular direction.

Single shot two-component velocity probability density functions (PDF) in the $\mathrm{H}_{2}$ - air flame at an equivalence ratio of $0.1(T=585 \mathrm{~K})$ are shown in Fig. 6. Data was extracted and processed from 200 single shot interferograms. The measured radial velocity component which is measured directly is $V_{r}=V_{1}=1 \pm 23 \mathrm{~m} / \mathrm{sec}$ where the uncertainty reported is \pm 1 standard deviation, and the velocity component calculated in axial direction, $V_{z}=8 \pm 82 \mathrm{~m} / \mathrm{sec}$. The average measured axial velocity is the same order of magnitude (a few $\mathrm{m} / \mathrm{s}$ ) with a simple estimate of the gas velocity in the flame based on the law of conservation of mass. With increased temperatures, the goodness of fit starts to decrease and implicitly the measurement uncertainty increases mainly because of a lower number of photons reaching the detector. For temperatures up to $1600 \mathrm{~K}$, the standard deviation of the radial component remains in the range of less than $40 \mathrm{~m} / \mathrm{sec}$, which is about $1 \%$ of the dynamic range of the instrument. For reasonable signal levels, at the expense of losing the spatial information, the average of measured velocities from two adjacent spectra from a single interferogram, or of all four spectra should improve the precision of measuring velocity by $\sqrt{2}$ and 2 , respectively, assuming that the errors are uncorrelated. With increasing temperature, an increasing bias of the measured velocity was found in the measurement. More elaborate modeling of the Rayleigh spectra beyond the classic Gaussian assumption might also improve these results. Another strategy that could improve the results includes increasing the angle between the Rayleigh detection angles to greater than 33 degrees, thereby improving the sensitivity to the axial velocity.

\section{Conclusions}

The combination of a dual-pump coherent anti-Stokes Raman scattering system with an interferometric Rayleigh scattering system (CARS-IRS) for the first time provided time-resolved simultaneous measurement of multiple properties in combustion flows. A spectrometer and a planar Fabry-Perot interferometer used in the imaging mode are employed to record the spectrally broad CARS spectra and the spontaneous Rayleigh scattering spectra, respectively. The velocity was determined by analyzing Fabry-Perot interferograms containing spectral as well as spatial information of the measurement volume. The technique was demonstrated by performing timeresolved simultaneous measurements of temperature, absolute mole fractions of $\mathrm{N}_{2}, \mathrm{O}_{2}$, and $\mathrm{H}_{2}$, and two components of velocity in a low velocity Hencken burner flame. Single pulse Rayleigh spectra were obtained from room temperature up to stoichiometric condition in the flame at $2380 \mathrm{~K}$.

With more elaborate software processing, and increase of the signal intensity ${ }^{11}$ the velocimetry technique described here will be capable of obtaining the velocity vector information at high temperature supersonic combustion flows required to improve CFD models. Translational temperature and gas density, computed from the spectra using the gas composition from CARS, can also be inferred from the same Rayleigh spectra. This would provide an important check on the measured CARS temperatures while providing the only measure of density. For example, the measurement of multiple temperatures along the CARS probe volume will allow the assumption of a single temperature in the CARS probe volume to be checked.

\section{Acknowledgments}

This work was supported by Mr. George Rumford, program manager of the Defense Test Resource Management Center's (DTRMC) Test and Evaluation/Science and Technology (T\&E/S\&T) program, under the Hypersonic Test focus area. We thank Lloyd Wilson for running the facility and Joe Lee for technical support during the optical setup. We also greatly acknowledge Noel Clemens at the University of Texas at Austin for first suggesting the combination of CARS and interferometric Rayleigh scattering techniques.

\section{References}

\footnotetext{
${ }^{1}$ Danehy, P.M., O’Byrne, S., Cutler, A.D., and Rodriguez, C. G., “Coherent anti-Stokes Raman Scattering (CARS) as a probe for supersonic hydrogen-fuel/air mixing,” JANNAF APS/CS/PSHS/MSS Joint Meeting, Colorado Springs, Colorado, December 1-5, 2003.
} 
2 O’Byrne, S., Danehy, P.M., Cutler, A.D., "Dual-Pump CARS Thermometry and Species Concentration Measurements in a Supersonic Combustor," $42^{\text {nd }}$ Aerospace Sciences Meeting and Exhibit, Reno, NV, January 5-8, 2004.

${ }^{3}$ Danehy, P. M., R. DeLoach, R., Cutler, A. D., "Application of Modern Design of Experiments to CARS Thermometry in a Supersonic Combustor," $22^{\text {nd }}$ AIAA Aerodynamic Measurement Technology and Ground Testing Conference, AIAA Paper 2002-2914, June 2002.

${ }^{4}$ Miles, B., Richard, Lempert, R., Walter and Forkey, N. Joseph, "Laser Rayleigh Scattering," Measurement Science and Technology, Vol. 12, pp. 33-51, 2001.

${ }^{5}$ Seasholtz, R. G., Zupanc, F. J. and Schneider, S. J., "Spectrally Resolved Rayleigh Scattering Diagnostics for Hydrogen-Oxygen Rocket Plume Studies,” J. Propulsion and Power, Vol. 8, No. 5, 1992, pp. 935-942.

${ }^{6}$ Seasholtz, R. G., Buggele, A. E. and Reeder, M., "Instantaneous Measurements in a Supersonic Wind tunnel Using Spectrally Resolved Rayleigh Scattering," Proceedings of the International Symposium on Optical Science, Engineering and Instrumentation, Society of Photo-Optical Instrumentation Engineers, Bellingham, WA, 1995.

${ }^{7}$ Bivolaru, D., Ötügen, M. V., Tzes, A. and Papadopoulos, G., "Image Processing for Interferometric Mie and Rayleigh Scattering Velocity Measurements, AIAA Journal, Vol. 37, No. 6, pp. 688-694, 1999.

${ }^{8}$ Bivolaru, D., Danehy, P. M., Lee, J. W., Gaffney, Jr. R. L., and Cutler, A. D., "Single-pulse Multi-point Multicomponent Interferometric Rayleigh Scattering Velocimeter," AIAA-2006-0836, 44th Aerospace Sciences Meeting, Reno, NV, January 9-12, 2006.

9 Seasholtz, R.G., Panda, J., "Rayleigh Scattering Diagnostic for Dynamic Measurement of Velocity and Temperature,” AIAA-99-0641, 37th Aerospace Sciences Meeting, Reno, NV, 1999.

${ }^{10}$ Seasholtz, R. G. \& Panda, J. "Rayleigh Scattering Diagnostic for Simultaneous Measurements of Dynamic Density and Velocity,” AIAA-2000-0642, 38th Aerospace Sciences Meeting, Reno, NV, January 2000.

${ }^{11}$ Bivolaru, D., Danehy, P. M., and Lee, J. W, "Intracavity Rayleigh-Mie Scattering for multipoint, two-component velocity measurement,” Optics Letters, Vol. 31, No. 11, pp. 1645-1647, June, 2006.

${ }^{12}$ A. Mielke, K. Elam, C. Sung, "Rayleigh Scattering Diagnostic for Measurement of Temperature, Velocity, and Density Fluctuation Spectra," 44th AIAA Aerospace Sciences Meeting and Exhibit, AIAA-2006-837, Reno, Nevada, January 9-12, 2006.

${ }^{13}$ J. W. Hahn, C. W. Park, and S. N. Park "Broadband coherent anti-Stokes Raman spectroscopy with a modeless dye laser", Applied Optics, Vol. 36, No. 27, 20 September 1997, p. 6722-6728.

${ }^{14}$ Eckbreth, A.C., Laser Diagnostics for Combustion Temperature and Species, $2^{\text {nd }}$ Ed., Gordon and Breach Pub., 1996.

${ }^{15}$ Palmer, R.E. The CARSFT Computer Code for Calculating Coherent Anti-Stokes Raman Spectra: User and Programmer Information, Sandia National Laboratories Report SAND89-8206, Livermore, California, 1989. 